\title{
ARTICLES
}

\section{New strategy for multi-colour fluorescence in situ hybridisation: COBRA: COmbined Binary RAtio labelling}

\author{
HJ Tanke ${ }^{1}$, J Wiegant ${ }^{1}$, RPM van Gijlswijk ${ }^{1}$, V Bezrookove $^{1}$, H Pattenier $^{1}$, \\ RJ Heetebrij ${ }^{2}, E G$ Talman $^{2}$, A K R aap ${ }^{1}$ and J V rolijk ${ }^{1}$ \\ ${ }^{1} \mathrm{~L}$ aboratory for Cytochemistry and Cytometry, D epartment of M olecular Cell B iology, L eiden U niversity M edical \\ Center \\ ${ }^{2} \mathrm{~L}$ eiden Institute of Chemistry, G orlaeus L aboratories, L eiden U niversity, L eiden, The N etherlands
}

\begin{abstract}
Multicolour in situ hybridisation (MFISH) is increasingly applied to karyotyping and detection of chromosomal abnormalities. So far 27 colour analyses have been described using fluorescently labelled chromosome painting probes in a so-called combinatorial approach. In this paper a new strategy is presented to use efficiently the currently available number of spectrally separated fluorophores in order to increase the multiplicity of M FISH . We introduce the principle of COBRA (CO mbined Binary RA tio labelling), which is based on the simultaneous use of combinatorial labelling and ratio labelling. $\mathrm{H}$ uman chromosome painting in $\mathbf{2 4}$ colours is accomplished using four fluorophores only. Three fluorophores are used pair wise for ratio labelling of a set of 12 chromosome painting probes. The second set of 12 probes is labelled identically but is also given a binary label (fourth fluorophore). The COBRA method is demonstrated on normal human chromosomes and on a lymphoma (J V M) cell line, using probes enzymatically labelled with fluorescein, lissamine and cy5 as primary fluorophores, and diethylaminocoumarin (DEAC), a blue dye, as combinatorial fourth label to demonstrate incorporated digoxigenin. In addition, the principle was tested using chemical labelling. T he first set of 12 painting probes was therefore labelled by U LS (U niversal L inkage System), using D E A C, cy3 and cy5 as primary labels, and the second set was labelled similarly, but also contained a digoxigenin-U LS label, which was indirectly stained with fluorescein. Subsequently, a mathematical analysis is presented and methods are indicated for achieving an MFISH multiplicity of $\mathbf{4 8 , 9 6}$ or even higher using existing technology.
\end{abstract}

Keywords: cytogenetics; chromosomal aberrations; multicolour FISH ; combinatorial labelling; chemical labelling; ratio labelling; digital fluorescence microscopy

Correspondence: Hans J Tanke PhD, Laboratory for Cytochemistry and Cytometry, Department of Molecular Cell $B$ iology, L eiden U niversity $M$ edical Center, Wassenaarseweg 72: $2333 \mathrm{~A} \mathrm{~L}$ Leiden, The N etherlands. Tel: 003171 5276196; Fax: 003171 5276180; E-mail: h.j.tanke@mcb.medfac . leidenuniv.nl

R eceived 2 J une 1998; revised 28 August 1998; accepted 7 September 1998 


\section{Introduction}

The introduction of fluorescence in situ hybridisation (FISH) has significantly changed cytogenetics. PCR amplification of DNA from human chromosomes obtained by flow sorting or by micro-manipulation allows generation of probe sets that specifically paint one type of chromosome or parts thereof. R ecently, multicolour FISH using 24 chromosome specific painting probes was used to produce a full FISH karyogram. ${ }^{1,2}$

FISH karyotyping is now successfully applied to elucidate complex chromosome rearrangements. ${ }^{3} \mathrm{Mul}$ ticolour FISH analysis of chromosomes is not necessarily restricted to the use of whole chromosome paints. Recently, sets of probes have been generated that specifically recognise the (sub)telomeric regions of a particular chromosome and are applied in a multicolour FISH format to detect cryptic translocations, frequently occurring in mental retardation. ${ }^{4} \mathrm{M}$ oreover, multicolour FISH using sub-regional DNA probes created by microdissection or isolated from human/ rodent somatic cell hybrids allows for artificially banded (bar coded) chromosomes. ${ }^{5,6}$ The number of bands in such FISH stained chromosomes depends on how many probes one may want to generate; there are, however, in principle, no limitations to recognising as many bands as are seen in Giemsa banded chromosomes (about 800).

The selective staining of 24 human chromosomes is at present accomplished by binary combinations of probes that are labelled with five distinct fluorophores. ${ }^{1,2,7}$ For this so-called combinatorial labelling (also called multiplex $\mathrm{FISH}^{2}$ ) the number of recognisable targets $(n)$ using (k) different fluorophores is $n=2^{k}-1$ colours. $^{8}$ Five fluorophores thus allow a maximum of 31 colours, sufficient to recognise 24 chromosomes, but insufficient for instance to explore the use of $p$ and $q$ arm specific probes for the detection of intrachromosomal rearrangements. Thus multicolour FISH analysis of chromosomes would benefit directly from a method of increasing the number of simultaneously recognisable targets beyond the 27 reported so far.

$\mathrm{H}$ igher FISH multiplicity using the binary approach is feasible by increasing the number of fluorophores to six. Since at present the available spectrum for five fluorophores is al ready utilised from the U V to the near infra-red, introduction of a sixth dye may lead to increased spectral overlap and undesirable cross talk between detection channels. More narrow excitation and emission filters may circumvent that problem, but inevitably lead to longer exposure times because of the reduced number of available photons. A Iternatively, higher FISH multiplicity is achievable by ratio labelling. This technique, by which a given probe is composed of a mixture of probes with different fluorescent labels, has great potential. A s an illustration, one may consider the number of recognisable colours that could be composed from the three primary colours blue, green and red. In practice, however, ratio labelling is considerably more complex than combinatorial labelling. Recognition of chromosomes stained with ratio labelled probes is not a 'yes or no' colour decision (as in the binary approach) but requires accurate measurement of colour.

The present paper explores the advantages of both methods, emphasising practical considerations and limitations. A strategy is described to achieve a FISH multiplicity of 48,96 or more. It is based on the strategic combination of binary labelling and ratio labelling, utilises existing technology and requires no major investment in microscope hardware other than a good digital fluorescence microscope.

\section{Materials and Methods}

\section{Principle of COBRA: COmbined Binary RAtio labelling}

The principle of COBRA is based on the simultaneous use of combinatorial (ie binary) labelling and so-called ratio labelling. ${ }^{9-11} A$ number of spectrally separated fluorophores is used for ratio labelling, in such a way that maximally two fluorophores are used to produce a certain colour. When this is applied for three fluorophores, and each pair of fluorophores results in five colours, a total of 12 colours is achieved (lower triangle in Figure 1). This primary probe set is directly fluorescently labelled using methods such as nick translation and labelling by DOP-PCR or chemical labelling. ${ }^{12,13} \mathrm{~A}$ second set of 12 probes, recognising different targets is labelled in exactly the same way, but in addition is given a hapten (for instance biotin or digoxigenin). This hapten is developed using avidin or antibodies labelled with a fourth fluorescent label, spectrally easily distinguishable from the three primary fluorophores used for ratio labelling. Thus, the set of 12 is multiplied by two, which results in 24 colours using four fluorophores only (two middle triangles in Figure 1), which is one fluorophore less than reported so far to accomplish staining of the 24 human chromosomes. Extra 'free' fluorophores may be used to repeat this process, exploring a second hapten, which again results in a doubling of the number of achievable colours (giving 48 colours) (upper triangles in Figure 1).

Obviously, even greater increments in the number of colours are achievable if more than 12 primary colours are produced in the basic triangle, either by using more than three fluorophores or by distinguishing more ratios. 


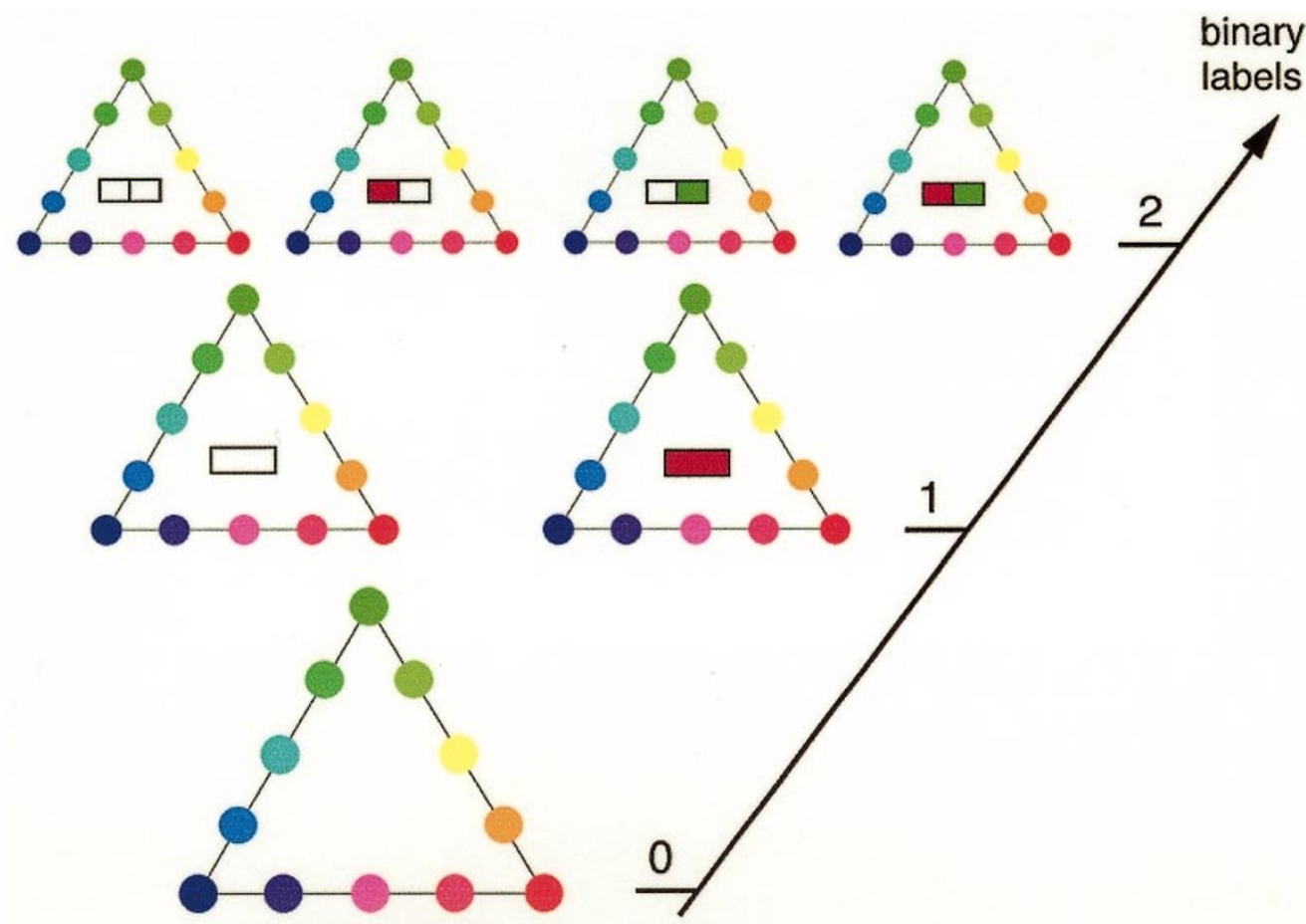

12 ratio colours

Figure 1 Principle of COBRA. The primary set of 12 ratio colours is doubled each time an independent binary label is introduced, resulting in 24 colours for 1 hapten, and in 48 colours for 2 haptens

$M$ athematically, the total number of achievable COBRA colours is described as follows. A ssume that $n$ fluorochromes are used for ratio labelling and only two of those fluorochromes are simultaneously used per target, while additionally $m$ fluorochromes can be binary labelled to the same target and $r$ ratios can be resolved for ratio labelling, then the number of different colours that can be distinguished is given by the following formula:

$$
\text { No. of colours }=\left(n+\frac{r \times n !}{2 !(n-2) !}\right) \cdot 2^{m}
$$

where

$$
\begin{aligned}
& \leq n \leq \infty \\
& \leq r \leq \infty \\
& \leq m \leq \infty
\end{aligned}
$$

\section{Examples:}

a. 2 fluorochromes for ratio labelling $(n=2), 0$ ratios $(r=0)$ and 0 binary label $(m=0)$ results in 2 colours, as expected;

b. 3 fluorochromes for ratio labelling $(n=3), 3$ ratios $(r=3)$ and 1 binary label $(m=1)$ results in 24 colours (the situation that will be demonstrated in this paper);

c. Increasing the number of ratios to $r=4$ and the number of fluorochromes for ratio labelling to 4 , results in 28 colours; d. Each binary fluorochrome results in doubling of the number of colours; that is to 56 (for 1) or to 112 (for 2).

The principle of this concept is demonstrated on 24 human chromosomes using enzymatic labelling of probes and probe mixing to accomplish ratio labelling, as well as direct attachment of the colour code to the probes using chemical labelling. For the enzymatic labelling we used fluorescein, lissamine and cy 5 as the three basic colours, and diethylaminocoumarin (DEAC), a blue fluorophore, as fourth fluorophore. For chemical labelling DEAC, cy3 and cy5 served as primary fluorophores, and fluorescein was used as the fourth binary label.

\section{Multicolour FISH Staining of Human Chromosomes}

Preparation of human metaphase chromosomes was performed as described by Wiegant et al. ${ }^{12,13}$ Chromosomes from normal human individuals as well as from in vitro cultured J V M-2 cells were used. ${ }^{14} \mathrm{Dr}$ N igel Carter (Sanger Institute, Cambridge, UK) kindly provided the probes for chromosomes 1-8, 13-20, 22 and $X$. In addition, probes for all chromosomes were obtained from Cytocell, A dderbury, B anbury, UK. A II probe DNA was amplified by DOP-PCR ${ }^{15}$ to generate a set of painting probes for all 24 human chromosomes. For the enzymatical labelling, approach probes for $1-8,13-20,22$ and $X$ from $\mathrm{Dr}$ Nigel Carter were supplemented with probes for chromosomes 9-12, 21 and $Y$ from Cytocell. For the chemical labelling approach only probes from Cytocell were used. 
Table 1

\begin{tabular}{rrrrr}
\hline $\begin{array}{c}\text { Chrom. } \\
\text { N o. }\end{array}$ & $\begin{array}{l}\text { Fluorescein } \\
(\%)\end{array}$ & $\begin{array}{c}\text { Lissamine } \\
(\%)\end{array}$ & $\begin{array}{r}\text { Cy 5 } \\
(\%)\end{array}$ & $\begin{array}{r}\text { D ig } \\
(\%)\end{array}$ \\
\hline 1 & 300 & 0 & 0 & 0 \\
7 & 150 & 50 & 0 & 0 \\
Y & 60 & 50 & 0 & 0 \\
19 & 85 & 150 & 0 & 0 \\
3 & 0 & 300 & 0 & 0 \\
9 & 0 & 75 & 40 & 0 \\
15 & 0 & 100 & 150 & 0 \\
21 & 0 & 70 & 150 & 0 \\
5 & 0 & 0 & 300 & 0 \\
11 & 35 & 0 & 80 & 0 \\
17 & 120 & 0 & 150 & 0 \\
13 & 150 & 0 & 75 & 0 \\
4 & 300 & 0 & 0 & 100 \\
10 & 160 & 40 & 0 & 100 \\
16 & 110 & 100 & 0 & 100 \\
22 & 80 & 150 & 0 & 100 \\
6 & 0 & 300 & 0 & 100 \\
12 & 0 & 80 & 35 & 100 \\
18 & 0 & 100 & 100 & 100 \\
$X$ & 0 & 90 & 150 & 100 \\
2 & 0 & 0 & 300 & 100 \\
20 & 80 & 0 & 150 & 100 \\
14 & 120 & 0 & 120 & 100 \\
8 & 150 & 0 & 50 & 100 \\
\hline
\end{tabular}

Note: $100 \%$ is equal to $100 \mathrm{ng}$ of labelled probe

Enzymatic Labelling of Probes A II probes were fluorescently labelled by incorporation of labelled dU TPs either by PCR or nick translation using fluorescein-, digoxigenin-dUTP (all from B oehringer $M$ annheim, G ermany), lissamine-dU TP (NEN Life Science Products, Boston, MA, USA) or cy5-dU TP (A mersham, A mersham, UK). The digoxigeninlabelled probes were detected indirectly using diethylaminocoumarin (DEAC) (M olecular Probes, E ugene, OR, USA). For labels and identification of the various probes see Table 1.

\section{Chemical Labelling of Probes using ULS (Universal}

Linkage System) Chemical labelling of probes was performed using the U niversal Linkage System (U L S) (K reatech D iagnostics, A msterdam, The Netherlands). DE A C, cy3 and cy5 were chosen as primary fluorophores and fluorescein as combinatorial fourth label to demonstrate digoxigenin-U LS (dig-U LS) labelled probes. ${ }^{16}$ The following strategy was used to label and dissolve the U LS-labelled probe set.

First, chromosome-specific painting probes for chromosomes $2,4,6,8,10,12,14,16,18,20,22$ and $X(100-400 \mathrm{ng})$ were labelled in one reaction with dig-U LS according to the manufacturers' instructions. Thereafter, this probe set was purified on a Q iagen quick spin column ( $Q$ iagen Inc, Valencia, CA , U SA ) according to the manufacturers' instructions. The labelled probe mixture was eluted from the Qiagen column using $100 \mu \mathrm{l}$ of $10 \mathrm{mM}$ Tris $\mathrm{HCl} \mathrm{pH} 8.5$.

Second, all chromosome-specific painting probes were fluorescently labelled according to Table 2 by mixing $30 \mu \mathrm{l}$ of the listed U LS compounds (or mixtures thereof) with $1 \mu \mathrm{g}$ of chromosome-specific painting probe D NA (all from Cytocell) using DEA C-ULS $(26.7 \mu \mathrm{M})$, cy3-ULS $(20 \mu \mathrm{M})$ and cy5-U LS
$(13.3 \mu \mathrm{M})$ in a final volume of $100 \mu$ of water. In case probes were labelled with mixtures of two different U L S compounds, the ULS compounds were first mixed in the desired ratio before the probe DNA was added. A fter 15 min incubation at $65^{\circ} \mathrm{C}$, the labelled probes were purified on Q iagen quick spin columns. The labelled probes were eluted from the Qiagen columns using $100 \mu \mathrm{l}$ of $10 \mathrm{mM}$ Tris H Cl pH 8.5. Prior to the hybridisation, fluorescent ULS-labelled probes were combined in amounts as indicated in the right-hand column of Table 1 together with the $100 \mu$ l of dig-U LS labelled probe mixture from the first step. This probe mixture was then ethanol-precipitated in the presence of $10 \times$ excess low molecular weight fish sperm DNA (Boehringer M annheim), and $3 \times$ excess human $\mathrm{C}_{0} \mathrm{tl}-\mathrm{D} N \mathrm{NA}$ (G ibco, BRL, Paisley, UK). Thereafter the probe mixture was dissolved in $10 \mu \mathrm{l} 50 \%$ deionised formamide, $2 \times \mathrm{SSC}, 50 \mathrm{mM}$ sodium phosphate

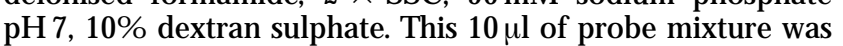
used as hybridisation solution (see later).

FISH Staining of Human Metaphase Chromosomes Hybridisation conditions of enzymatically and ULS labelled probes were similar. Slides with metaphase chromosomes were pretreated with $\mathrm{R} N$ ase A and pepsin according to Wiegant et al. ${ }^{17}$ The chromosome preparations were denatured by incubating them for $90 \mathrm{sec}$ at $80^{\circ} \mathrm{C}$ in $60 \%$ formamide, $2 \times \mathrm{SSC}, \mathrm{pH} 7$ on a hotplate. A fter removal of the coverslip the slides were dehydrated through an ethanol series and air dried. Then, $10 \mu$ l hybridisation mixture was applied under a $18 \times 18 \mathrm{~mm}$ coverslip, sealed with rubber cement, and hybridisation was performed for $120 \mathrm{hr}$ at $37^{\circ} \mathrm{C}$ in a humid chamber. In case enzymatically labelled probes were used, the hybridisation mixture contained $50 \%$ formamide, $2 \times$ SSC, $50 \mathrm{mM}$ sodium phosphate $\mathrm{pH} \mathrm{7,} 10 \%$ dextran

Table 2

\begin{tabular}{|c|c|c|c|c|}
\hline $\begin{array}{l}\text { Chrom. } \\
\text { No. }\end{array}$ & $\begin{array}{l}\mu \mathrm{DLEAC}- \\
\mathrm{ULS} \\
(26.7 \mu \mathrm{M})\end{array}$ & $\begin{array}{l}\mu \mathrm{ll} \text { Сy3- } \\
U L S \\
(20 \mu \mathrm{M})\end{array}$ & $\begin{array}{l}\mu \text { l Cy5- } \\
\text { ULS } \\
(13.3 \mu \mathrm{m})\end{array}$ & $\begin{array}{l}\text { ng probe } \\
\text { DNA } \\
\text { in hybrid. mix }\end{array}$ \\
\hline 1 & 30 & 0 & 0 & 500 \\
\hline 2 & 0 & 0 & 30 & 300 \\
\hline 3 & 0 & 30 & 0 & 400 \\
\hline 4 & 30 & 0 & 0 & 500 \\
\hline 5 & 0 & 0 & 30 & 600 \\
\hline 6 & 0 & 30 & 0 & 500 \\
\hline 7 & 22.5 & 9 & 0 & 300 \\
\hline 8 & 22.5 & 0 & 9 & 400 \\
\hline 9 & 0 & 22.5 & 7.5 & 500 \\
\hline 10 & 22.5 & 9 & 0 & 500 \\
\hline 11 & 7.5 & 0 & 22.5 & 500 \\
\hline 12 & 0 & 22.5 & 7.5 & 400 \\
\hline 13 & 22.5 & 0 & 9 & 300 \\
\hline 14 & 19.5 & 0 & 15 & 300 \\
\hline 15 & 0 & 18 & 15 & 300 \\
\hline 16 & 15 & 18 & 0 & 300 \\
\hline 17 & 19.5 & 0 & 15 & 300 \\
\hline 18 & 0 & 18 & 15 & 400 \\
\hline 19 & 7.5 & 22.5 & 0 & 400 \\
\hline 20 & 7.5 & 0 & 22.5 & 400 \\
\hline 21 & 0 & 10.5 & 22.5 & 300 \\
\hline 22 & 7.5 & 22.5 & 0 & 400 \\
\hline$X$ & 0 & 10.5 & 22.5 & 400 \\
\hline Y & 15 & 18 & 0 & 100 \\
\hline
\end{tabular}



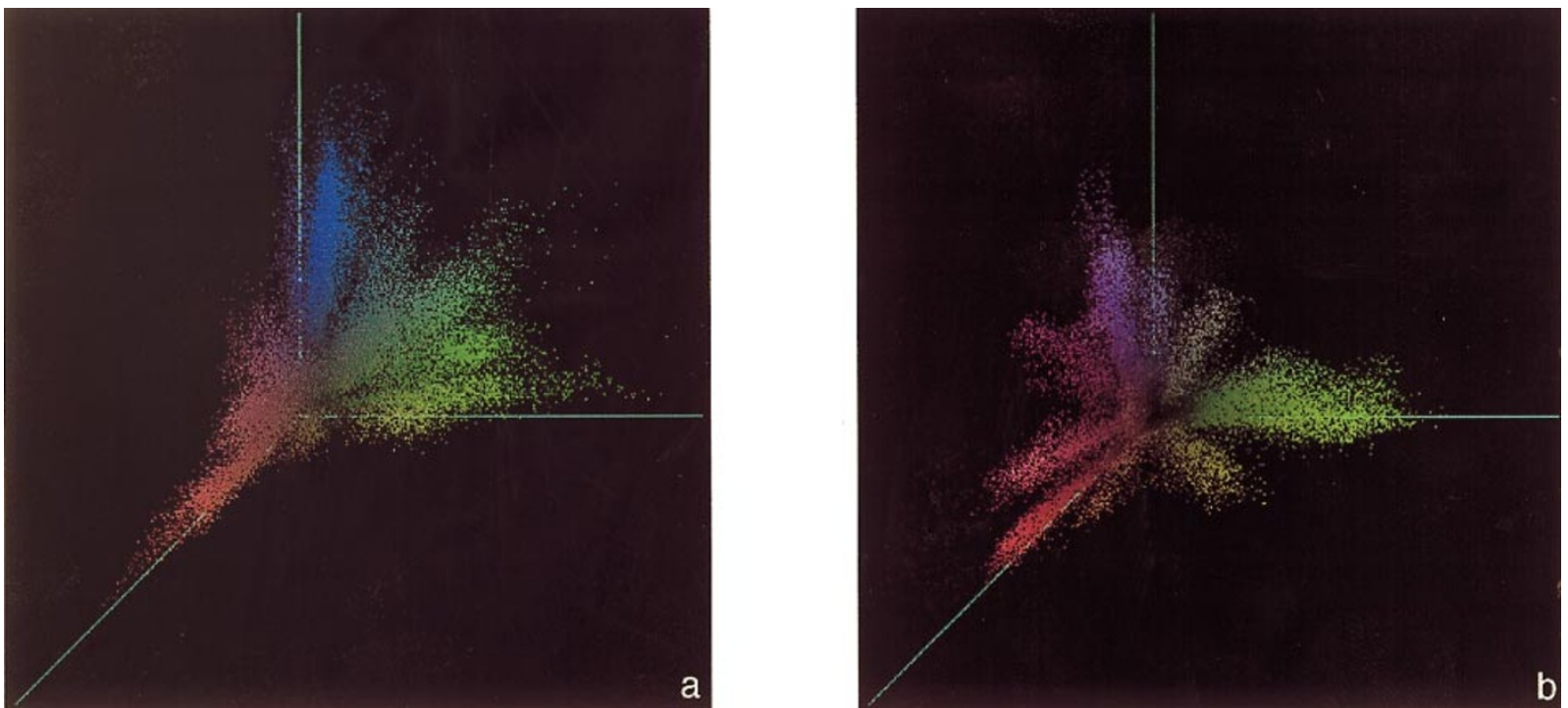

Figure 2 Human chromosomes were stained in 24 colours using the COBRA principle. For each of the 24 chromosomes the fluorescence intensity was plotted in a three-dimensional colour space. E ach coloured dot represents the measured colour intensity of an image point (pixel) of a certain chromosome. (a): three primary colours (fluorescein, lissamine, cy5); without binary DE AC label; (b): idem, with binary DEAC label.

N ote: Figure 1 is a schematic top view of the $2 \times 12$ clusters seen at equal $x, y, z$ values of the measured data shown in this figure

sulphate, $300 \mathrm{ng}$ of each fluorescein-, lissamine- and cy5-labeled probe (single labelled probes), 100-250 ng of each ratio labelled probe, $100 \mathrm{ng}$ of each digoxigenin labelled probe, $3 \times$ excess human $C_{0}$ tl-DNA and $10 \times$ excess low molecular weight fish sperm DNA in $10 \mu \mathrm{l}$. When chemically modified probes were used, the hybridisation mixture contained $50 \%$ formamide, $2 \times \mathrm{SSC}, 50 \mathrm{mM}$ sodium phosphate $\mathrm{pH} 7,10 \%$ dextran sulphate, 100-500ng of each DEA C-, cy3and cy5-labelled probe (both single and ratio labelled probes) (see Table 2), 100-400 ng of each dig-ULS labelled probe, $3 \times$ excess human $\mathrm{C}_{0} \mathrm{tl}-\mathrm{DNA}$ and $10 \times$ excess low molecular weight fish sperm DNA in $10 \mu \mathrm{l}$. Before application, the probes were denatured for $10 \mathrm{~min}$ at $80^{\circ} \mathrm{C}$, followed by $60 \mathrm{~min}$ incubation at $37^{\circ} \mathrm{C}$ to allow pre-annealing with the $3 \times$ excess of $C_{0}$ tl-D NA.

A fter a $10 \mathrm{~min}$ post-hybridisation wash in $2 \times \mathrm{SSC} / 0.1 \%$ Tween 20 at $37^{\circ} \mathrm{C}$ to remove the coverslips, the slides were washed $2 \times 5 \mathrm{~min}$ in $50 \%$ formamide, $2 \times \mathrm{SSC}$, pH 7 at $44^{\circ} \mathrm{C}$. This was followed by 2 washes ( $5 \mathrm{~min}$ each) in $0.1 \times \mathrm{SSC}$ at $60^{\circ} \mathrm{C}$ and a $5 \mathrm{~min}$ wash at RT in TNT $(0.1 \mathrm{M}$ Tris $\mathrm{HCl} \mathrm{pH} \mathrm{7.4,}$ $0.15 \mathrm{M} \mathrm{NaCl}, 0.05 \%$ Tween 20). The enzymatically labelled dig probes were detected with a mouse monoclonal antibody against digoxin (Sigma) followed by a sheep anti-mouse antibody conjugated to DEAC. The chemically labelled dig probes were detected with a mouse monoclonal antibody against digoxin (Sigma) followed by a rabbit anti-mouse antibody conjugated to FITC (Sigma, St Louis, USA). Chromosomes were counterstained with DA PI. The slides were embedded in Vectashield (when enzymatically labelled probes were used) or Citifluor (A gar, Stansted, U K) (when chemically labelled probes were used) prior to microscopical evaluation.

\section{Digital Imaging Microscopy}

D igital fluorescence imaging was performed using a Leica DM-RXA epifluorescence microscope (Leica, Wetzlar, Germany) equipped with a 100-W mercury arc lamp and computer controlled filter wheels with excitation and emission filters for visualisation of DEAC, fluorescein, lissamine, cy3 and cy5, using H Q -FITC, Pinkel set plus SP 570, H Q -Cy3, H Q -Cy5 and DEA C filter (Chroma Technology, B rattleboro, $\mathrm{VT}, \mathrm{U} S \mathrm{~S}$ ) respectively. DA PI was excited with U V light using block A. A $63 \times$ objective (N.A. 1.32, PL A PO, L eica) was used. Image acquisition and analysis was performed on a Cytovision workstation (A pplied Imaging, Newcastle, UK). This system consists of a PC (Pentium $133 \mathrm{MHz}$ processor, $24 \mathrm{M} \mathrm{b} \mathrm{Ram}, 2.1 \mathrm{G} \mathrm{b}$ disc and 17 in display) interfaced to a Coolview camera (Photonic Science, Robertsbridge, UK). The camera has thermo-electric cooling, which allows on chip integration up to circa $30 \mathrm{sec}$. I mages are digitised in an 8-bit $768 \times 512$ image format.

Image acquisition was performed as described before. ${ }^{11}$ Chromosomes were segmented interactively by thresholding the DA PI image. The segmented image was used as a mask for the colour image, which was composed of the three images corresponding with the ratio labelled fluorochromes (green for fluorescein, red for lissamine and blue for cy5) and of the $D E A C$ image. Note that this procedure does not require thresholding of the three colours. The fourth DEAC image was evaluated binary, that is chromosomes with or without DEA C fluorescence were distinguished. This was performed by finding the optimal threshold in the histogram of the DEAC image for the pixels lying within the DAPI mask. Typically, two gaussion distributions were observed, corresponding to DEAC positive and negative chromosomes. 

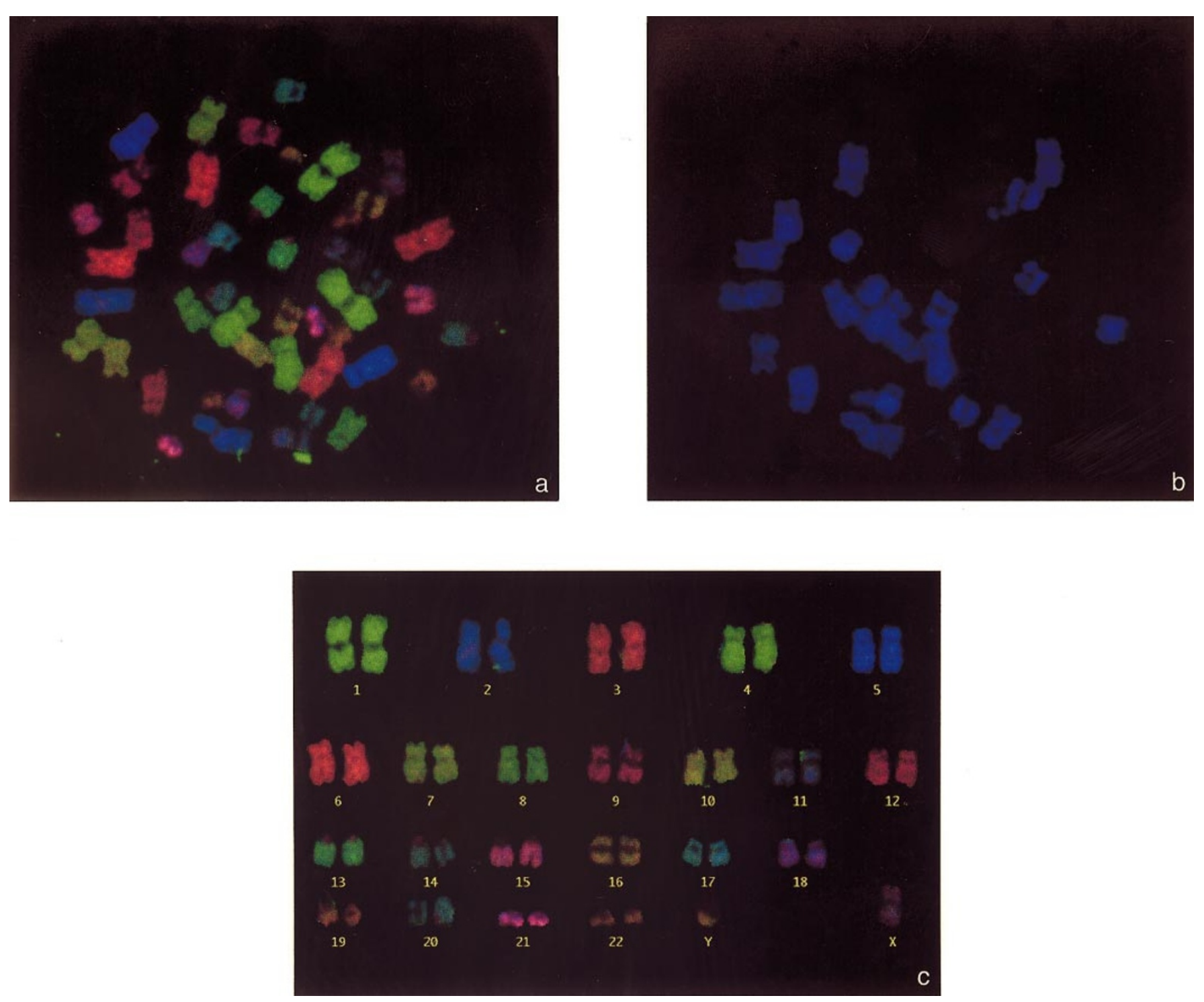

Figure 3 Normal human chromosomes labelled by COBRA in 24 colours (same data as Figure2). (a) I mage (12 colours) resulting from the primary dyes used in ratio labelling; (b) DEAC image of the same metaphase cell; (c) Karyogram resulting from the combination of image (a) and (b) and automated classification

Samples stained with ULS labelled probes were analysed similarly, in the sense that the ratio images related to DEAC (green), cy3 (red) and cy5 (blue), and the binary fourth image to fluorescein.

Classification was performed in two steps: the chromosome classification was followed by a pixel classification to detect possible translocations. Chromosome classification was based mainly on the modal colour value of each chromosome, eg its position in one of the colour triangles (the one with or without the binary label), as shown in Figure 1. The shortest distance of the measured modal colour value of a chromosome to the theoretical expected ratio colour of all chromosome classes was therefore calculated. In order to compensate for non-specific fluorescence contributions and to increase the robustness of the method the theoretical expected colour values were warped on to a triangle formed by the measured modal values of the chromosomes with only one ratio colour. B esides the modal colour value the length of the chromosomes was also used for classification. Theoretically, the colour values of the chromosomes should correspond to the original probe ratios. In practice, however, a more robust approach is obtained, when a number of metaphases is used for training the classifier (V rolijk et al, manuscript in preparation). Following object classification, each pixel within a chromosome was classified on the basis of the shortest distance to the measured chromosome classes. The binary (fourth colour) information of each pixel was used to decide within which colour triangle distance calculations should be 
performed. A ssignment of classification colours is considered useful and foreseen, but was not implemented in the current software.

Finally, a karyogram was generated based on chromosome classification showing the ratio colours, as described above. A karyogram, in which a pseudo colour was assigned to the corresponding chromosome class of each separate chromosome pixel was produced to facilitate the interactive detection of chromosome translocations. When needed the DAPI banding image was used for comparison purposes.

\section{Results}

A 24 colour COBRA staining procedure using four fluorophores was applied to normal and abnormal chromosomes. The optimal conditions for labelling the probes and the final composition of the probe set required some fine tuning due to the fact that some probes performed better than others. Typically, lower performing FISH probes were given colour combinations such that colour overlap with other probes was minimised.

O ptimal staining results were obtained at prolonged hybridisation times ( 5 days), although 3 days in many cases was sufficient. The use of $C_{0} t$ l-D NA was found essential for selective staining of chromosomes; the optimal amount per incubation mixture was a $3 \times$ excess.

Figure 2 shows how the 24 chromosomes occupy the colour space. Typically, within a certain chromosome image, signal intensities showed relatively large varia- tions, due to local differences in FISH intensity. The characteristic colour, however, was sufficiently constant to form clusters, with a defined angle within the 3-D colour space (Figure 2). A lthough some chromosome clusters showed overlap, they were well enough separated to be classified automatically using the procedure described above.

Figure 3 shows the actual chromosome images and the resulting karyogram. Integration times varied depending on the fluorophore used and ranged from 0.5 to $20 \mathrm{sec}$. A n entire COBRA acquisition and analysis procedure typically took approximately $1 \mathrm{~min}$. A pplied to abnormal chromosomes as shown in the JVM cell line, COBRA allowed for easy detection of abnormal chromosomes (Figure 4). The results shown in Figures 2-4 were obtained with enzymatically labelled probes, whereby ratio labelling was accomplished by mixing probes in desired proportions. The same COBRA principle was then applied to probes that were labelled chemically using ULS. In this method it is essential that in principle each probe molecule contains the ratio code, making mixing obsolete. A Ithough U LS modifications of fluorescein, rhodamine and cy5 performed adequately for single colour chromosome staining, stable ratios were not achieved. This is probably due to strongly different reaction kinetics of the ULS derivatives, and/or to physical interactions of the DNA bound dye molecules. Ratio labelling of DEAC, cy3 and cy5 performed much better, and could
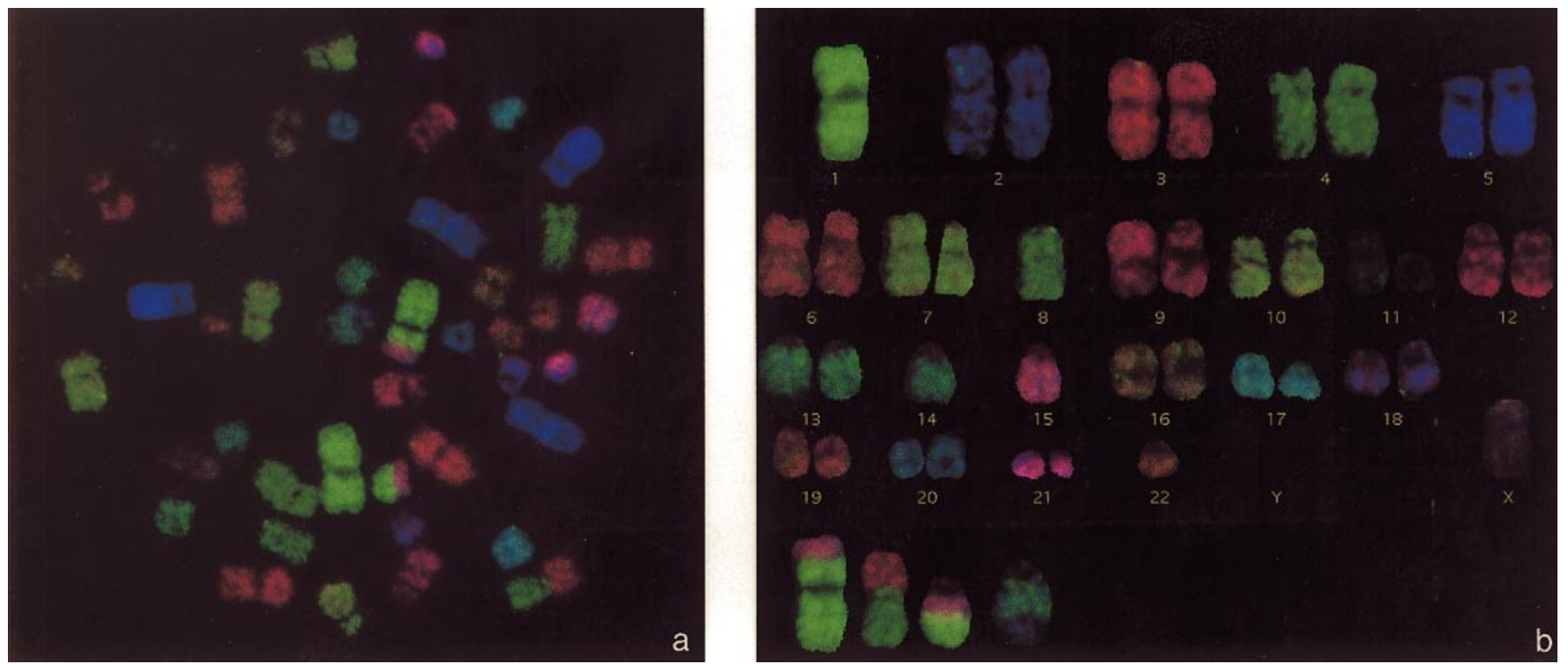

Figure 4 COBRA (24 colours) applied to a JVM cell line (B-prolymphocytic leukemia) showing translocations $t(11,14), t(3,8)$ and $\mathrm{t}(1,15)$ 
be well combined with binary fluorescein labelling. Results obtained with these probes are shown in Figure 5.
The robustness of COBRA depended on the quality of the metaphase chromosomes obtained, as is the case for automated analysis of both Giemsa-banded and
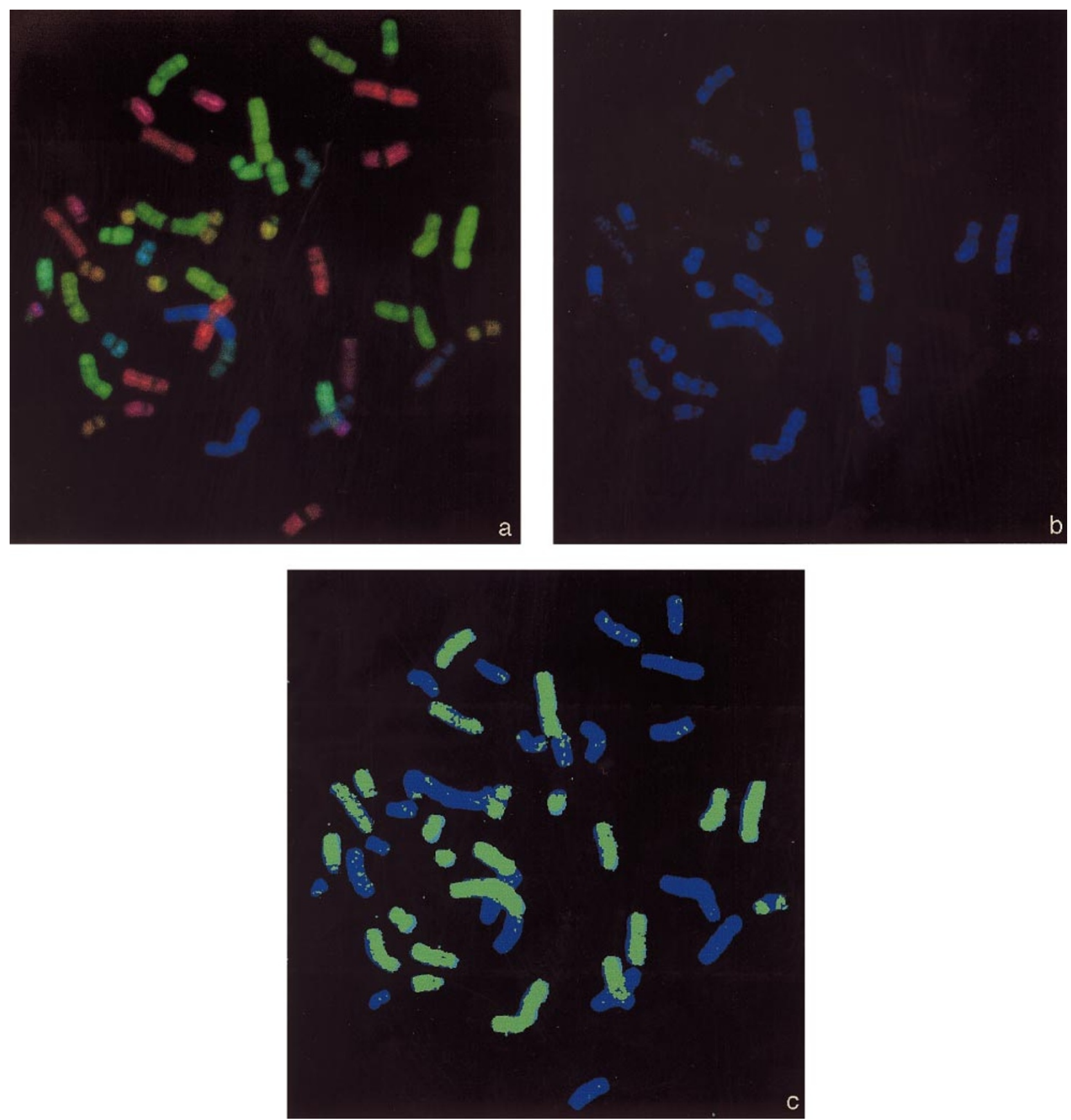

Figure 5 Results of ratio labelling obtained using chemical labelling (UL S system). The fluorophores D E AC, cy3 and cy5 were used as primary labels for ratio labelling. The dig-ULS labelled second set of 12 probes was demonstrated indirectly using fluorescein labelled immunoconjugates. (a) Image (12 colours) resulting from the three primary dyes used for ratio labelling; (b) Image of the binary (fourth) label (fluorescein, but shown in blue false colour); (c) Thresholded image (b) to identify the fluorescein positive and negative sets of chromosomes: N ote: DEAC is used as direct probe label here (by UL S), whereas in Figures 2 , and 4 it was used as binary label (as immunoconjugate) 
FISH -stained chromosomes. G ood quality slides always resulted in images of good signal-to-noise ratio that could be classified automatically, whereas user intervention increased with decreasing staining quality.

\section{Discussion}

D espite its only recent introduction, multicolour FISH karyotyping has changed cytogenetics significantly. Most studies reported so far use five different fluorophores in a combinatorial approach, which allows for a theoretical maximum of 31 colours.

A s has been shown, colours may also be created by mixing the same kind of probes, but now in different proportions to create various ratios of two or more kinds of fluorophores. ${ }^{6,12}$ These studies have shown that six or seven ratios of two different fluorophores can be distinguished. Ratio labelling therefore has great potential to increase the number of colours. H owever, its applications so far are limited, due to the fact that recognition of combinatorially labelled chromosomes is based on thresholding of the signal only (binary decision), whereas ratio labelled chromosomes require accurate measurements. If colours are created by mixing differently labelled probes, the variation in signal intensity and/or colour may be large due to variations in hybridisation efficacy as a function of the type of label. This could be overcome by avoiding mixing differently labelled probes and providing each D NA molecule with the various labels, achievable using enzymatic or chemical labelling procedure. A problem that may occur is the physical interaction of the different fluorophores bound to the same DNA molecule, causing energy transfer or quenching, which may result in poorly reproducible labelling. These potential problems cause M FISH so far to be based on combinatorial labelling using mixtures of probes.

The COBRA principle described in this paper combines the advantages of both approaches. It 'settles for' making ratios of two fluorophores only, but utilises the possibility of doubling the number of colours by introducing indirectly labelled haptens, which require a binary decision only. A s shown here, this approach is feasible and allows for identifying 24 human chromosomes using four fluorophores only. M oreover, the use of Cy7 an infra-red fluorescing dye is avoided, which allows for the use of less expensive cameras. The full potential of this approach has not yet been explored. So far only painting probes have been used in COBRA. Considering the short exposure times, we anticipate that other types of probe such as YA Cs or PA Cs can be used in a similar approach.

A s the mathematical equation shows, the number of colours particularly increases if more dyes or more ratios are used for the primary colour set. It has been shown that distinction of a ratio of 6 or 7 of two dyes is feasible. ${ }^{10}$

We anticipate that such an approach is best achieved if chemical labelling is used. Chemical labelling methods, such as U LS, may be advantageous for large scale production of quality controlled painting probes. Further exploration of multicolour FISH analysis in cytogenetics seems at present to depend more on the availability of quality controlled probes than on further development of FISH microscope workstations. A Ithough some refinement is needed, currently available hardware and software is well suited for M FISH . In this context the COBRA strategy for efficient use of fluorophores can significantly contribute to a further increase of M FISH multiplicity and thereby to further exploitation in cytogenetics.

\section{Acknowledgements}

This work was supported financially by the EUREKA program (SPE CTR A KA R ) of A pplied I maging Corporation, N ewcastle, UK and $K$ reatech Diagnostics, A msterdam, The N etherlands.

\section{References}

1 Schroeck E, du Manoir S, Veldman T et al: Multicolor spectral karyotyping of human chromosomes. Science 1996; 273: 494-497.

2 Speicher M R, B allard SG, Ward D C: K aryotyping human chromosomes by combinatorial multi-fluor FISH. N at G enet 1996; 12: 368-375.

3 Veldman T, Vignon C, Schrock E, Rowley J D, Ried T: $\mathrm{H}$ idden chromosome abnormalities in haematological malignancies detected by multicolour spectral karyotyping. $N$ at $\mathrm{G}$ enet 1997; 15: 406-410.

$4 \mathrm{~N}$ ing $\mathrm{Y}$, R oschke A, Smith ACM et al: A complete set of human telomeric probes and their clinical applications. $\mathrm{N}$ at $\mathrm{G}$ enet 1996; 14: 86-89.

5 Lengauer C, Speicher M R, Popp S et al: Chromosomal bar codes constructed by fluorescence in situ hybridization with A lu-PCR products of multiple Y A C clones. $\mathrm{H} \mathrm{um} \mathrm{M}$ ol G enet 1993; 2: 505-512.

6 Mueller S, R occhi M, Ferguson-Smith MA, Wienberg J: Towards a multicolour chromosome bar code for the entire human karyotype by fluorescence in situ hybridization. H um G enet 1996; 110: 271-278.

7 Nederlof PM, van der Flier S, Wiegant J et al: M ultiple fluorescence in situ hybridization. Cytometry 1990; 11: 126-131. 
8 Ried T, Baldini A, R and TC, Ward DC: Simultaneous visualization of seven different DNA probes by in situ hybridization using combinatorial fluorescence and digital imaging microscopy. Proc Nat Acad Sci USA 1992; 89: 1388-1392.

9 D auwerse J G, Wiegant J, R aap A K, Breuning M H, van Ommen GJB: Multiple colors by fluorescence in situ hybridization using ratio-labelled DNA probes create a molecular karyotype. H um M ol G enet 1992; 1: 593-598.

10 Morrison LE, Legator MS: Two-color ratio-coding of chromosome targets in fluorescence in situ hybridization: quantitative analysis and reproducibility. Cytometry 1997; 27: 314-326.

11 Nederlof PM, van der Flier S, V rolijk J, Tanke HJ, R aap A K : Fluorescence ratio measurements of double-labelled probes for multiple in situ hybridization by digital microscopy. Cytometry 1992; 13: 839-845.

12 Wiegant J, Ried T, N ederlof PM, van der Ploeg $M$, Tanke HJ, Raap A K: In situ hybridization with fluoresceinated D NA . Nucleic A cids Res 1991; 19: 3237-3241.

13 Wiegant J, Wiesmeijer CC, Hoovers J MN et al: M ultiple and sensitive fluorescence in situ hybridization with rhodamine-, fluorescein-, and coumarin-labeled DNA s. Cytogenet Cell G enet 1993; 63: 73-76.
14 M elo JV, B rito-Bapapulle V, Foroni L et al: Two new cell lines from B-prolymphocytic leukemia: characterization by morphology, immunological markers, karyotype and Ig gene rearrangement. Int J Cancer 1986; 38: 531-538.

15 Telenius $\mathrm{H}, \mathrm{C}$ arter N P, B epp CE, N ordenskjold M, Ponder $B A$, Tunnacliffe A: Degenerate oligonucleotide-primed PCR : general amplification of target DNA by a single degenerate primer. G enomics 1992; 13: 718-725.

16 Van B elkum A, L inkels E, Jelsma T, H outhoff HJ, Van den Berg FM, Q uint W: A pplication of a new, universal D NA labeling system in the PCR mediated diagnosis of Chlamydia trachomatis and human papilloma virus type 16 infection in cervical smears. J Virol Methods 1993; 45: 189-200.

17 Wiegant J, Galjart NJ, R aap A K, d'A zzo A: The gene encoding human protective protein (PPG $B$ ) is on chromosome 20. Genomics 1991; 10: 345-349. 